\title{
Interposition of the Anterior Tibial Tendon between the Medial and Intermediate Cuneiforms in Lisfranc Injuries: Report of Two Cases
}

\author{
Dong Q, Qu Z, Wang $G$ and Huang $\mathrm{H}^{*}$ \\ Department of Hand and Foot Surgery, Affiliated \\ Hospital of Qingdao University, Huangdao Area, \\ Qingdao, Shandong, People's Republic of China \\ *Corresponding author: Heng Huang, Department \\ of Hand and Foot Surgery, Affiliated Hospital of Qingdao \\ University, Huangdao Area, Qingdao, Shandong, People's \\ Republic of China
}

Received: June 28, 2021; Accepted: July 20, 2021;

Published: July 27, 2021

\begin{abstract}
Total lateral incongruity type Lisfranc injury may cause skin problems and compartment syndrome, manual reduction and temporary immobilization are usually needed. However, sometimes-manual reduction is not easy to achieve due to the prevention of reduction by interposed soft tissues. We report two cases of irreducible Lisfranc injuries due to interposition of the anterior tibial tendon between the medial and intermediate cuneiform bones. Our recommendation for manual reduction in total lateral incongruity with suspicion of the anterior tibial tendon's interposition is to push up the forefoot dorsally under the distraction, abduct the forefoot, displace the forefoot medially, and adduct the forefoot in sequence.
\end{abstract}

Keywords: Lisfranc injury; Interposition; Anterior tibial tendon; Manual reduction

\section{Introduction}

The Lisfranc injury has been well described and classified [1-3]. If the Lisfranc injury is not promptly diagnosed and properly treated, it can have devastating results $[2,4,5]$.

Manual reduction of the dislocated tarso-metatarsal joints and temporary immobilization with splints are usually needed because of concerns related to skin problems and compartment syndrome [6]. However, sometimes manual reduction is not easy to achieve due to the prevention of reduction by interposed soft tissues $[7,8]$.

We report two cases of irreducible Lisfranc injuries due to interposition of the anterior tibial tendon between the medial and intermediate bones.

\section{Case Presentation}

\section{Case 1}

A 47-year-old male sustained a vehicle accident and injured his left calf with an open wound, and his midfoot was injured. On physical examination, the external fixator was applied on his leg, and the dorsomedial aspect of the midfoot was prominent and moderately tender with ecchymosis. Plain radiographs showed tibial and fibular shaft comminuted fractures and total lateral incongruity type Lisfranc injury (type A according to Myerson's classification) (Figure 1).

Initially, he underwent irrigation and debridement of the open wound at the fracture site, and an external fixator was applied for tibial stabilization in another hospital. They also tried to manually reduce Lisfranc injury several times, but failed.

Thereafter, he was transferred to our hospital. We attempted manual reduction again under regional anesthesia and fluoroscopy, but also failed. We performed open reduction with the patient under spinal anesthesia. With a longitudinal incision from the talo-

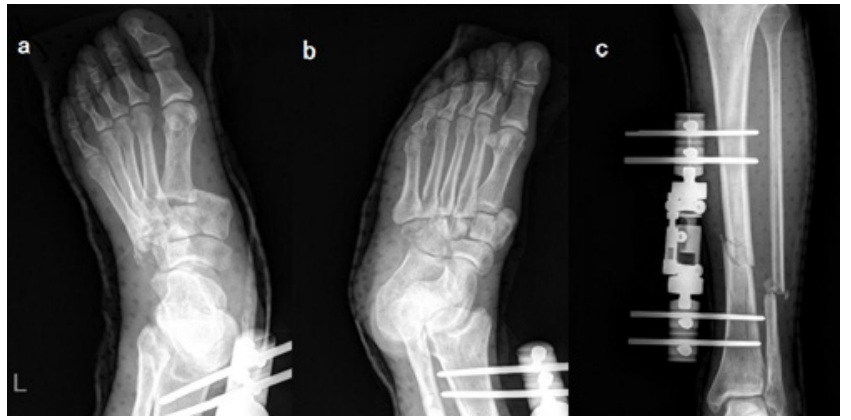

Figure 1: $a, b$ ) Show the total lateral incongruity Lisfranc injury (according to Myerson); c) Shows a tibial and fibular shaft comminuted fracture fixed with external fixation.

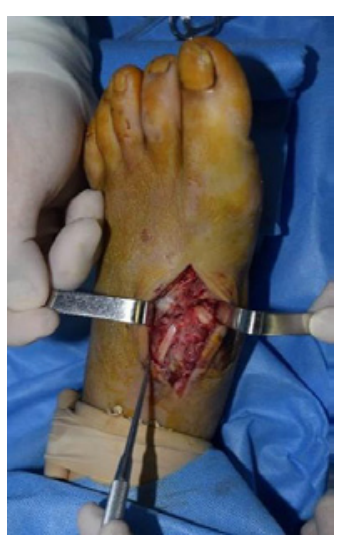

Figure 2: The anterior tibial tendon was interposed between the medial and intermediate cuneiform bones.

navicular joint to the middle $1 / 3$ of the first metatarsal, we exposed the dislocated first and second metatarsals and found the anterior 


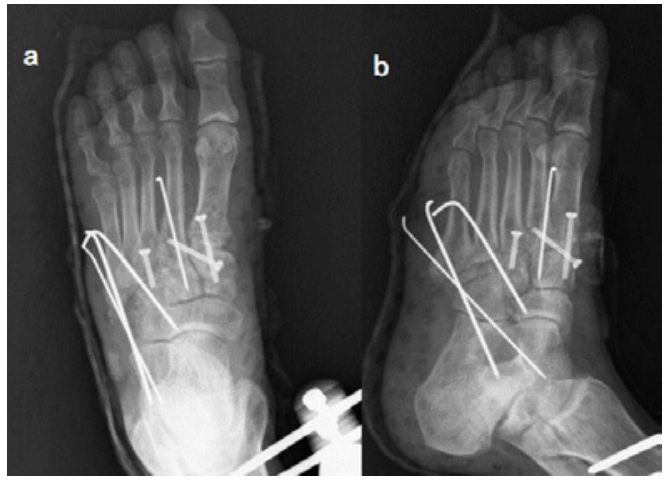

Figure 3: Postoperative AP and oblique X-rays show the Lisfranc joint fixed with 3 cannulated screws and $4 \mathrm{~K}$-wires.

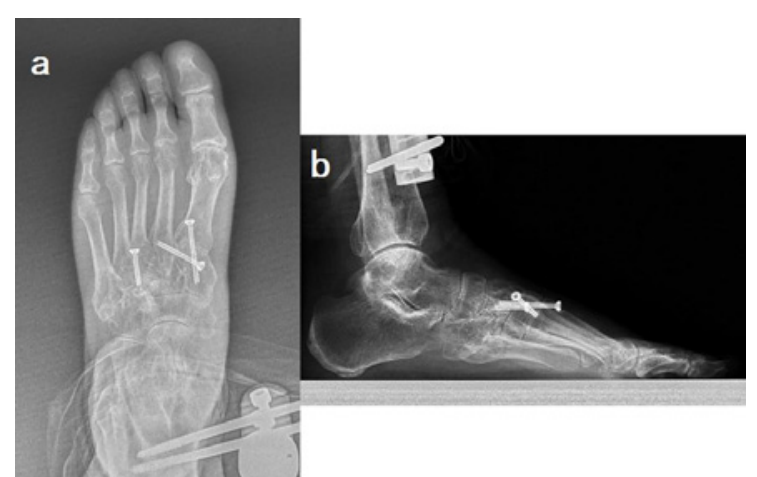

Figure 4: Postoperative 18-month $\mathrm{AP}$ and lateral $\mathrm{X}$ rays show that the Lisfranc joint alignment was normal with degenerative changes in the tarsometatarsal joints.

tibial tendon trapped between the medial and intermediate cuneiform bones (Figure 2). After the anterior tibial tendon was released, the reduction of the tarso-metatarsal joint was easily performed. The first and third tarso-metatarsal joints and the second metatarso-medial cuneiform joint were fixed with 4.0-mm cannulated screws (Carefix", China). Each 0.045-inch K-wire was used for fixation of the second and fifth tarso-metatarsal joints, and each 0.062-inch K-wire was used for fixation of the fourth and fifth tarso-metatarsal joints (Figure 3).

Postoperatively, we recommended active range of motion exercise after the pain subsided. All K-wires were removed at postoperative week 6, and tolerable weight bearing was allowed at 12 weeks. At 18 months postoperatively, the VAS (Visual Analogue Scale) score was 1 and the AOFAS (American Orthopedic Foot and Ankle Society)midfoot score was 100 . Postoperative 18-month X-rays showed that the Lisfranc joint alignment was normal, with slight degenerative changes in the tarso-metatarsal joints (Figure 4) and no tenderness. The power of the anterior tibial muscle was normal. There were no complaints about Lisfranc injury.

\section{Case 2}

A 20-year-old male had a right foot crush injury from a motor vehicle accident. On physical examination, he had a $5 \mathrm{~cm}$ skin wound on the medial aspect of the midfoot, and the forefoot was deformed. Plain radiographs revealed a total lateral incongruity type Lisfranc injury (type A according to Myerson's classification) with $2^{\text {nd }}, 3^{\text {rd }}$, and

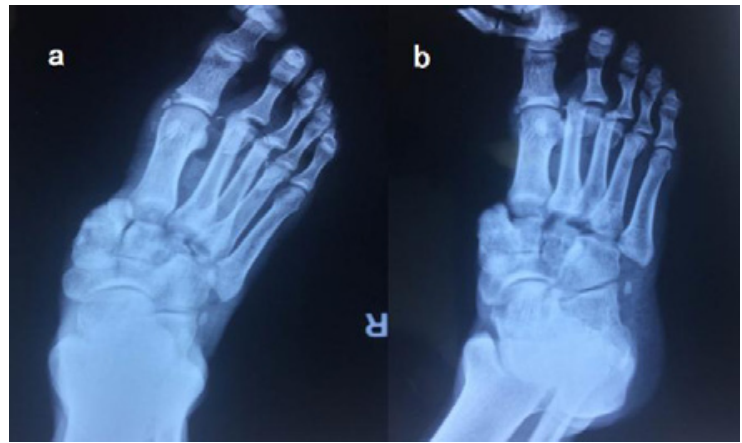

Figure 5: $a$ and $b$ show the total lateral incongruity Lisfranc injury with the $2^{\text {nd }}$ $3^{\text {rd }}$, and $4^{\text {th }}$ metatarsal head fractures and avulsion fracture of the proximal phalanx of the big toe.

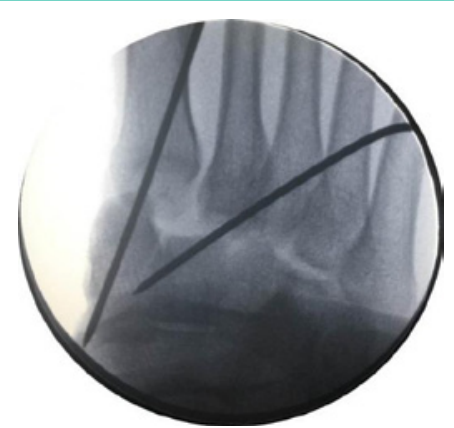

Figure 6: Incomplete reduction of the Lisfranc joint fixed with $2 \mathrm{~K}$-wires seen under intra-operative fluoroscopy.

$4^{\text {th }}$ metatarsal head fractures and avulsion fracture of the proximal phalanx of the big toe (Figure 5).

We performed wound debridement and manual reduction with plantar flexion and medial displacement of the forefoot under the regional anesthesia. However, the reduction was hard to maintain, so it was temporally fixed with 0.062 -inch K-wire (Figure 6). Two weeks later, we performed open reduction. Intraoperatively, we observed that the anterior tibial tendon was split and that the lateral half was trapped between the medial and intermediate cuneiforms. It was blocking the reduction of the Lisfranc injury (Figure 7). After the interposed lateral half of the anterior tibial tendon was released, anatomic reduction was achieved. The tarso-metatarsal joints were fixed with multiple 0.062 -inch K-wires because of the open wound, and the second, third, and fourth metatarsal heads were each fixed with cannulated screws (Carefix', China) (Figure 8). The postoperative rehabilitation protocol was the same as that for case 1 .

At 12 postoperative months, the VAS (visual analogue scale) score was 0 and the AOFAS (American Orthopedic Foot and Ankle Society)-midfoot score was 92 . The open wound healed, and the power of the anterior tibial muscle was normal. There was no complaint about Lisfranc injury.

\section{Discussion}

Most Lisfranc injuries result from a crushing injury to the dorsum of the foot. These high-energy injuries may result in severe soft tissue disruption in the midfoot and various patterns of dislocation of the tarso-metatarsal joints. Myerson et al. [4] classified Lisfranc 


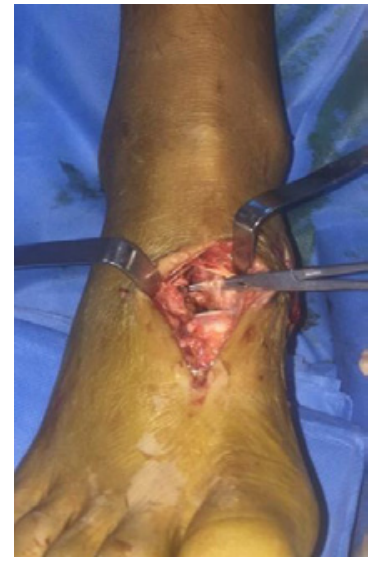

Figure 7: The lateral slip of the anterior tibial tendon was interposed between the medial and intermediate cuneiform bones.

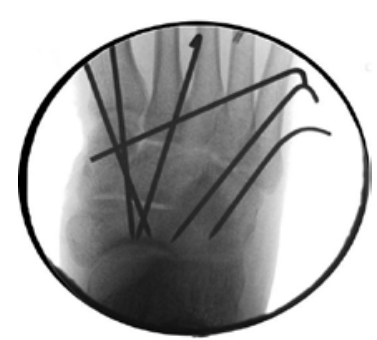

Figure 8: The Lisfranc injury was fixed with multiple K-wires.

injuries in detail depending on the radiographic findings [5]. This classification divided injuries in terms of joint congruity, location of the involvement, and the direction of instability. According to this classification, our two cases had total lateral incongruity type Lisfranc injury.

Meanwhile, in a comprehensive study of 290 feet, Hallisy [9] reported that $90 \%$ of the anterior tibial tendon was bifid at $1-2 \mathrm{~cm}$ proximal to the tarso-metatarsal joint. The medial slip of the anterior tibial tendon inserts into the medial and inferior surfaces of the medial cuneiform and the lateral slip to the base of the first metatarsal. So the first metatarsal is displaced laterally, and the lateral slip of the anterior tibial tendon is pulled laterally with the base of the first metatarsal, whereas the medial slip remains in position [4]. If there is instability between the medial and intermediate cuneiform bones, the lateral slip of the anterior tibial tendon may become interposed between the medial and intermediate cuneiform bones [7,10-13]. Involuntary contraction of the tibialis anterior muscle at the time of injury may also contribute to the interposition of the anterior tibial tendon between the medial and intermediate cuneiform bones [4].

However, we consider that the interposition of lateral slip of the anterior tibial tendon between the medial and intermediate bones is caused not only by the lateral displacement of the first metatarsal at the time of Lisfranc injury but also by the traction of the dorsally and laterally displaced first metatarsal during the manual reduction in total lateral incongruity Lisfranc injury. The anterior tibial tendon is usually displaced dorsally and laterally with the first metatarsal and located above the tarsal bones in total lateral incongruity Lisfranc injury. So when we perform the manual reduction, we usually distract and push the first metatarsal plantarly to reduce the dorsally and laterally displaced metatarsal bones. If this is combined with instability of the medial-intermediate cuneiform joint at the time of injury, the tensioned lateral slip of the anterior tibial tendon may be trapped within the medial-intermediate cuneiform joint during manual reduction.

Therefore, our recommendation for manual reduction in total lateral incongruity with suspicion of the anterior tibial tendon's interposition is to push up the forefoot dorsally under the distraction, abduct the forefoot, displace the forefoot medially, and adduct the forefoot in sequence. This sequence of manual reduction can prevent the interposition of the anterior tibial tendon between the medial and intermediate cuneiform bones. Furthermore, the dislocated metatarsals had self-reduction power, which was observed during open reduction after releasing the interposed anterior tibial tendon.

\section{References}

1. Hardcastle $\mathrm{PH}$, Reschauer R, Kutscha-Lissberg $\mathrm{E}$, Schoffmann W. Injuries to the tarsometatarsal joint. Incidence, classification and treatment. J Bone Joint Surg Br. 1982; 64: 349-356.

2. Myerson $M$. The diagnosis and treatment of injuries to the Lisfranc joint complex. Orthop Clin North Am. 1989; 20: 655-664.

3. Quénu E, Kuss G. Etude sur les luxations du metatarse (luxationsmetatarsotarsiennes) du diastasis entre le 1er et le 2e metatarsien. Rev Chir. 1909; 39: 1093-1134.

4. Myerson MS, Fisher RT, Burgess AR, Kenzora JE. Fracture dislocations of the tarsometatarsal joints: end results correlated with pathology and treatment. Foot Ankle. 1986; 6: 225-242.

5. Philbin T, Rosenberg G, Sferra JJ. Complications of missed or untreated Lisfranc injuries. Foot Ankle Clin. 2003; 8: 61-71.

6. Lattermann C, Goldstein JL, Wukich DK, Lee S, Bach BR, Jr. Practical management of Lisfranc injuries in athletes. Clin J Sport Med. 2007; 17: 311 315.

7. Blair WF. Irreducible tarsometatarsal fracture-dislocation. J Trauma. 1981; 21: $988-990$

8. Lowe J, Yosipovitch Z. Tarsometatarsal dislocation: a mechanism blocking manipulative reduction. Case report. J Bone Joint Surg Am. 1976; 58: 10291030.

9. Hallisy JE. The muscular variations in the human foot. A quantitative study. General results of the study: I. Muscles of the inner border of the foot and the dorsum of the great toe. Dev Dyn. 1930; 45: 411-442

10. Karaindros K, Arealis G, Papanikolaou A, Mouratidou A, Siakandaris P. Irreducible Lisfranc dislocation due to the interposition of the tibialis anterior tendon: case report and literature review. Foot Ankle Surg. 2010; 16: e68-71.

11. Ashworth MJ, Davies MB, Williamson DM. Irreducible Lisfranc's injury: the 'toe up' sign. Injury. 1997; 28: 321-322.

12. Denton JR. A complex Lisfranc fracture-dislocation. J Trauma. 1980; 20: 526529.

13. Engber WD, Roberts JM. Irreducible tarsometatarsal fracture-dislocation. Clin Orthop Relat Res. 1982; 168: 102-104. 